\title{
POWER BOUNDED RESTRICTIONS OF FOURIER-STIELTJES TRANSFORMS
}

\author{
ROGER ANDERSSON
}

\section{Introduction.}

In the present paper we give local variants of the theorems of BeurlingHelson and Cohen, i.e. characterizations of the power bounded elements in certain restriction algebras of Fourier-Stieltjes transforms on locally compact abelian groups. It is well known that there are no obvious such characterizations in general. However, we will provide a characterization, presented in some detail below, in the case when the algebra consists of restrictions to a set $E$ which is compact and which is the closure of its interior. We also obtain some results in the case where $E$ is the closure of an open connected set.

Let $G$ be a locally compact abelian group with dual group $\Gamma$ amd let $M(\Gamma)$ be the convolution algebra of all bounded regular Borel measures $\mu$ on $\Gamma$ such that $\|\mu\|_{M(\Gamma)}$, the total variarion of $\mu$, is finite. The Fourier-Stieltjes transform of $\mu \in M(\Gamma)$ is defined by

$$
\hat{\mu}(x)=\int_{\Gamma} \overline{\langle x, \gamma\rangle} d \mu(\gamma) .
$$

For a closed set $E$ in $G$ we denote by $B(E)$ the algebra of restrictions of Fourier-Stieltjes transforms of elements in $M(\Gamma)$ to $E$. With the norm defined by

$$
\|f\|_{B(E)}=\inf \left\{\|\mu\|_{M(\Gamma)} \mid \hat{\mu}=f \text { on } E\right\},
$$

$B(E)$ is a Banach algebra of continuous functions. The set of all functions $f \in B(E)$ with $|f|=1$ on $E$ and $\sup _{n \in Z_{+}}\left\|f^{n}\right\|_{B(E)}<\infty$ will be denoted by $\mathscr{B}(E)$. By the well known theorem of Beurling and Helson [1], for $G$ connected, any function in $\mathscr{B}(G)$ is a character times a complex constant of modulus one. A more general result due to Cohen, (See [4, Theorem 1], [12, Theorem 4.7.3]) gives a characterization of the functions in $\mathscr{B}(G)$, valid for any $G$. The main result of this paper is a similar characterization of the functions in $\mathscr{B}(E)$ when $E$ is compact and the closure of its interior. We will now describe this result.

Received May 30, 1979. 
Let $T$ be the group of complex numbers of modulus one and $U$ an open neighborhood of the identity in $G$. A continuous map $\theta: U \rightarrow \mathrm{T}$ is called a local character if whenever $x \in U, y \in U$ and $x+y \in U$ then $\theta(x+y)=\theta(x) \theta(y)$. If $\bar{U}$ is compact then the continuous extrapolation to $\bar{U}$ of $\theta$ is in $\mathscr{B}(\bar{U})$ (cf. Section 2). We denote by $\mathscr{L}(E)$ the set of functions that locally on $E$ agree with translates of local characters multiplied by complex numbers of modulus one. When $E$ is compact, it follows by a partition of the unit that $\mathscr{L}(E) \subset \mathscr{B}(E)$. Our characterization is that if $E$ is compact and the closure of its interior then $\mathscr{B}(E)=\mathscr{L}(E)$. For such sets we also give a more explicit description of the functions in $\mathscr{L}(E)$ (cf. Section 5).

We begin by proving that if $V$ is any open set in $G$ and $f \in \mathscr{B}(\bar{V})$ then $\left.f\right|_{V} \in \mathscr{L}(V)$. This is done by structure theory using the above-mentioned result of Cohen and a local version of the theorem of Beurling and Helson [3] in $\mathbf{R}^{n}$. Using a technique introduced by Domar in [4] we then improve this result, showing that for some integer $p$ not exceeding $\sup _{n \in Z_{+}}\left\|f^{n}\right\|_{B(\bar{V}),} f$ agrees locally on $V$ with translates of just $p$ essentially different local characters multiplied by complex numbers of modulus one. This gives a natural partition of $V$ in $p$ open subsets $V_{k}, f$ being locally equal to a translate of one and the same local character times a complex number of modulus one on $V_{k}$. Then, if $x$ is a boundary point of some $V_{k}$ we choose a small neighborhood $W$ of the identity in $G$ with $\bar{W}$ compact and divide $f$ by the $x$-translate of the local character. For sufficiently small $W$, the quotient is in $\mathscr{B}\left(\overline{V_{k} \cap(x+W)}\right)$ and is locally constant in $V_{k} \cap(x+W)$. The proof is finished by showing that such a function has a finite range.

In Section 6 we first give a few examples which show that there is no simple general characterization of the functions in $\mathscr{B}(E)$, when $E$ is the closure of an open set. However, if $E$ is the closure of an open and connected set, the functions in $\mathscr{B}(E)$ admit a simple description, similar to that given by the theorem of Beurling and Helson (cf. Section 6). The section ends with a discussion of for which sets $E$, functions of this kind are in $\mathscr{B}(E)$. For a positive result when $E$ is non-compact and disconnected we refer to Svensson [13].

I wish to thank Docent Philip Brenner for suggesting the topic and for his kind interest in my work. I also wish to express my gratitude to Professor Yngve Domar, whose work in the area of harmonic analysis has been a great source of inspiration. In some of the work on the problems discussed here I benefited from time spent at the Mittag-Leffler Institute during the academic year 1973-1974 and in the spring of 1977.

\section{Preliminaries.}

We begin by reviewing certain notions concerning topological groups. Any 
group considered in this paper, unless the contrary is explicitly stated, will be locally compact and abelian (abbreviated LCA). With some obvious exceptions like $T$, the group of complex numbers of modulus one, all groups will be written additively with 0 as identity element.

A homomorphism is said to be a morphism if continuous and an epimorphism if it is continuous, open and surjective. If $U$ is an open neighborhood (shortened open nbd) of 0 in $G$, a continuous map $\theta$ of $U$ into a group $H$ is called a local morphism if whenever $x \in U, y \in U$ and $x+y \in U$ then $\theta(x+y)$ $=\theta(x)+\theta(y)$. A local isomorphism of $G$ with $H$ is a homeomorphism $\theta$ of a nbd of 0 in $G$ onto a nbd of 0 in $H$ such that $\theta$ and $\theta^{-1}$ are local morphisms. When such a map exists the groups are said to be locally isomorphic. Morphisms (local morphisms) into $T$ are called characters (local characters).

In general a local character is not a restriction of a character. However the following device (cf. eg. [10, Theorem 18]) enables us to regard it as induced by a character of a "larger" group.

THEOREM 2.1. Let $\theta: U \rightarrow \mathrm{T}$ be a local character in $G$, let $G_{0}$ be the open subgroup of $G$ generated by $U$ and let $\tilde{U}=\{(x, \theta(x)) \in G \times T \mid x \in U\}$. Let $\tilde{G}$ be the subgroup of $G \times \mathrm{T}$ generated by $\tilde{U}$ and (disregarding its topology as a subgroup of $G \times T)$ topologized by taking as a base for nbds of the origin the sets $\tilde{V}=\{(x, \theta(x)) \in G \times \mathrm{T} \mid x \in V \subset U\}, V$ ranging over the open nbds of 0 in $G$. If $\alpha$ and $\beta$ are the restrictions to $\tilde{G}$ of the projection of $G \times \mathrm{T}$ onto $G$ and of the projection of $G \times T$ onto $T$, respectively, then

(1) $\widetilde{G}$ is LCA

(2) $\alpha: \tilde{G} \rightarrow G_{0}$ is an epimorphism and $\left.\alpha\right|_{\tilde{U}}: \tilde{U} \rightarrow U$ is a homeomorphism, whence $\tilde{G}$ and $G$ are locally isomorphic

(3) $\beta$ is a character of $\tilde{G}$ and $\theta=\beta \circ\left(\left.\alpha\right|_{\tilde{U}}\right)^{-1}$.

Remark 1. The group $\tilde{G}$ is minimal in the following sense: If (1), (2) and (3) above are satisfied with $\tilde{G}, \tilde{U}, \alpha$ and $\beta$ changed to $G^{\prime}, U^{\prime}, \alpha^{\prime}$ and $\beta^{\prime}$ respectively, then $\widetilde{G}$ is isomorphic with an open subgroup of $G^{\prime}$ modulo a discrete subgroup.

Remark 2. Let $\alpha: R^{q} \rightarrow R^{q} / Z^{q}=T^{q}$ be the canonical map, $U$ an open connected nbd of 0 in $R^{q}$ contained in $]-\frac{1}{4}, \frac{1}{4}\left[{ }^{q}\right.$ and $V=\alpha(U)$. For any $a \in \mathbf{R}^{q}$

$$
\theta_{a}: x \rightarrow e^{2 \pi i\left\langle a,(\alpha \mid U)^{-1}(x)\right\rangle}, \quad x \in V,
$$

is a local character on $V\left(\langle\right.$,$\left.\rangle is the usual inner product in \mathrm{R}^{q}\right)$. Conversely any local character on $V$ is of this form, since a local morphism in $R^{q}$ coincides on some nbd of 0 with a morphism [2, p. 88]. It is readily seen that in this case $\tilde{R}^{q}$ 
is (isomorphic with) $\mathrm{R}^{q} / \mathrm{Z}^{a} \cap(a)^{\perp}$, where $(a)^{\perp}$ denotes the annihilator of the subgroup generated by $a$.

In Section 3 we will need a certain type of basis at 0 in compact abelian groups. Much more general results of this type are known [10, Theorem 69], but for the convenience of the reader we include a direct proof of the special case we will use.

THEOREM 2.2. For each nbd $U$ of 0 in a compact abelian group $G$ there is a closed subgroup $H$, a morphism $\psi: \mathbf{R}^{q} \rightarrow G$ for some $q \geqq 0\left(\right.$ with $\left.\mathbf{R}^{0}=\{0\}\right)$ and an open connected $\mathrm{nbd} V$ of 0 in $\mathrm{R}^{q}$ such that

(1) $\psi(V)+H$ is open and contained in $U$

(2) the map $(t, h) \rightarrow \psi(t)+h,(t, h) \in V \times H$ is a local isomorphism.

Proof. Since the dual group $\Gamma$ of $G$ is discrete there are $\gamma_{1}, \ldots, \gamma_{p}$ in $\Gamma$ and $\varepsilon>0$ such that $U$ contains the open set

$$
N(p, \varepsilon)=\left\{x \in G||\left\langle x, \gamma_{j}\right\rangle-1 \mid<\varepsilon, 1 \leqq j \leqq p\right\} .
$$

The subgroup $\Lambda$ of $\Gamma$ generated by $\gamma_{1}, \ldots, \gamma_{p}$ is isomorphic with a quotient group of $Z^{p}$ and can thus be written as a direct sum, $\Lambda=\Lambda_{1} \oplus \Lambda_{2}$ where $\Lambda_{1}$ is isomorphic with $Z^{q}$ and $\Lambda_{2}$ is a finite group. We may assume that $\varepsilon$ is so small that $N(p, \varepsilon) \subset \Lambda_{2}^{\perp}$, the annihilator of $\Lambda_{2}$. Let $H=\Lambda^{\perp}$. If $q=0$ then $H=N(p, \varepsilon)$ and (1) and (2) are trivially satisfied. If $q>0$ we may also assume that $\gamma_{1}, \ldots, \gamma_{q} \in \Lambda_{1}$, that $\gamma_{q+1}, \ldots \gamma_{p} \in \Lambda_{2}$ and that for a suitable isomorphism $\sigma: \Lambda_{1} \rightarrow Z^{q}, \sigma\left(\gamma_{j}\right)=e_{j}$, where $e_{j}$ is the element in $Z^{q}$ whose $j$ th coordinate is 1 and the others are 0 . Let $\tau: Z^{q} \rightarrow R^{q}$ be the inclusion map. As $\mathbf{R}^{q}$ is divisible and $\Gamma$ is discrete, we can extend $\tau \circ \sigma$ to a morphism $\varrho$ of $\Gamma$ into $\mathrm{R}^{q}$. Let $\psi: \mathrm{R}^{q}$ $\rightarrow G$ be the dual of $\varrho$, i.e.

$$
\forall \gamma \in \Gamma \forall t \in \mathbf{R}^{q}\langle\psi(t), \gamma\rangle=e^{2 \pi i\langle\boldsymbol{Q}(\gamma), t\rangle},
$$

and let

$$
V=\left\{t=\left(t_{1}, \ldots, t_{q}\right) \in \mathbf{R}^{q}|| t_{j} \mid<\frac{1}{\pi} \arcsin \frac{\varepsilon}{2}, 1 \leqq j \leqq q\right\} .
$$

Now if $\varepsilon$ is small and $x \in N(p, \varepsilon)$, there is a unique $t=\left(t_{1}, \ldots, t_{q}\right) \in V$ such that $\left\langle x, \gamma_{j}\right\rangle=e^{2 \pi i t} j$ for $1 \leqq j \leqq q$ and thus, $x-\psi(t) \in \Lambda_{1}^{\perp}$. But $x-\psi(t) \in \Lambda_{2}^{1}$ too, since $N(p, \varepsilon) \subset \Lambda_{2}^{1}$ and $\varrho\left(\Lambda_{2}\right)=\{0\}$. Hence $N(p, \varepsilon)=\psi(V)+H$ and the map $(t, h)$ $\rightarrow \psi(t)+h,(t, h) \in V \times H$ is a homeomorphism, as the argument above also shows that its continuous extension to the compact $\bar{V} \times H$ is injective. 
Remark. If $q=0$ for each nbd $U$ of 0 in $G$, then $G$ is totally disconnected and conversely.

In our notations we will in general follow [12] but with the roles of $G$ and $\Gamma$ reversed, thus taking Fourier and Fourier-Stieltjes transforms on $G$ of functions and measures on $\Gamma$. We will frequently use the following immediate consequence of [12, Lemma 4.2.2].

LeMma 2.1. If $\alpha$ is a morphism of the group $G_{1}$ into the group $G_{2}$, if $E \subset G_{2}$ is closed and $f \in B(E)$ then $f \circ \alpha \in B\left(\alpha^{-1}(E)\right)$ and $\|f \circ \alpha\|_{B\left(\alpha^{-1}(E)\right)}\|f\|_{B(E)}$.

The next result, due to de Leeuw and Herz [5], enables us to go in the opposite direction.

THEOREM 2.3. If $\alpha$ is a morphism of the group $G_{1}$ into the group $G_{2}$, if $E \subset G_{1}$ is compact and $\alpha$ is injective on a nbd of E, then there is a constant $C$ such that if $f \in B(E)$ then $f \circ\left(\left.\alpha\right|_{E}\right)^{-1} \in B(\alpha(E))$ and $\left\|f \circ\left(\left.\alpha\right|_{E}\right)^{-1}\right\|_{B(\alpha(E))} \leqq C\|f\|_{B(E)}$.

In particular this theorem and Theorem 2.1. imply that if $U$ is an open nbd of 0 with $\bar{U}$ compact, the continuous extrapolation to $\bar{U}$ of a local character on $U$ is in $\mathscr{B}(\bar{U})$.

For a closed set $E$ in $G$ we denote by $A(E)$ the algebra of restrictions to $E$ of Fourier transforms of functions in $L^{1}(\Gamma)$. Thus $f \in A(E)$ if there is a $\varphi \in L^{1}(\Gamma)$ such that

$$
f(x)=\hat{\varphi}(x)=\int_{\Gamma} \overline{\langle x, \gamma\rangle} \varphi(\gamma) d \gamma, x \in E
$$

and

$$
\|f\|_{A(E)}=\inf \left\{\|\varphi\|_{L^{1}(\Gamma)} \mid \hat{\varphi}=f \text { on } E\right\} .
$$

We finally remind the reader that if $E$ is compact and $\varepsilon>0$ there is a $k \in L^{1}(\Gamma)$ with $\hat{k}=1$ on $E$ and $\|k\|_{L^{1}(\Gamma)}<1+\varepsilon$. Thus for compact sets $E$, we have $B(E)$ $=A(E)$ and with the same norms.

\section{A local result.}

Let $E$ be a closed set in a LCA group $G$ and $\mathscr{B}(E)$ the set of all functions $f \in B(E)$ with $|f|=1$ on $E$ and $\sup _{n \in Z_{+}}\left\|f^{n}\right\|_{B(E)}<\infty$. Though our main results on $\mathscr{B}(E)$ require $E$ to be compact or connected, in this and the next section we only assume $E$ to be a closed set with a non-empty interior.

THEOREM 3.1. If $f \in \mathscr{B}(E)$ then each interior point in $E$ has a nbd in which $f$ equals a translate of some local character times a constant of modulus one. 
The essential step in the proof is the following lemma.

Lemma 3.1. Let $W$ be an open connected nbd of 0 in $\mathrm{R}^{p}$, and $H$ a compact group. If $\mathrm{g} \in \mathscr{B}(\bar{W} \times H)$ then there is a piecewise affine map $C: H \rightarrow T$ and a piecewise constant map $a: H \rightarrow R^{p}$ such that

$$
g(t, h)=C(h) e^{2 \pi i\langle a(h), t\rangle}, \quad(t, h) \in \bar{W} \times H .
$$

We follow [12] in the definitions and properties of affine and piecewise affine maps. For the convenience of the reader, we repeat these definitions here.

Let $G_{1}$ and $G_{2}$ be LCA groups and $E$ a coset in $G_{2}$, i.e. a translate of a subgroup of $G_{2}$. If $\alpha: E \rightarrow G_{1}$ is a continuous map such that

$$
\alpha\left(x+x^{\prime}-x^{\prime \prime}\right)=\alpha(x)+\alpha\left(x^{\prime}\right)-\alpha\left(x^{\prime \prime}\right), \quad x, x^{\prime}, x^{\prime \prime} \in E,
$$

then $\alpha$ is said to be affine. If $S_{i}, 1 \leqq i \leqq m$, are pairwise disjoint sets belonging to the coset ring of $G_{2}$, each $S_{i}$ contained in an open coset $K_{i}$ of $G_{2}$ and $\alpha_{i}: K_{i}$ $\rightarrow G_{1}$ is affine for each $i$, then the map $\alpha$ of $\bigcup_{i=1}^{m} S_{i}$ into $G_{1}$ which coincides on $S_{i}$ with $\alpha_{i}$ is said to be piecewise affine. The coset ring of $G_{2}$ is the smallest ring of subsets of $G_{2}$ containing all open cosets in $G_{2}$. Piecewise constant maps are defined similarly with obvious modifications.

Proof of the lemma. First fix $h \in H$ and consider the function $g_{h}$ : $t \rightarrow g(t, h)$ on $\bar{W}$. As the map $t \rightarrow(t, h), t \in \mathrm{R}^{p}$, is affine, $g_{h} \in \mathscr{B}(\bar{W})$ by Lemma 2.1. By [3, Theorem 1], which is correct for $\Gamma=\mathrm{R}^{n}$, there are $C(h) \in \mathrm{T}$ and $a(h) \in \mathbf{R}^{p}$ such that

$$
g_{h}(t)=C(h) e^{2 \pi i\langle a(h), t\rangle}, \quad t \in \bar{W},
$$

thus defining the maps $C$ and $a$. On the other hand, for $t \in \bar{W}$ fixed, the function $g_{t}: h \rightarrow g(t, h)$ is in $\mathscr{B}(H)$, again by Lemma 2.1. By the result of Cohen (cf. [4, Theorem 1], [12, Theorem 4.7.3]) the function $g_{t}$ is piecewise affine. Choosing $t=0$ we see that $C$ is piecewise affine, hence so are all the functions $h \rightarrow e^{2 \pi i\langle a(h), t\rangle}$. This implies $a$ to be piecewise affine. But a piecewise affine map from a compact group into $R^{p}$ is piecewise constant, since the only compact subgroup of $R^{p}$ if $\{0\}$.

Remark. If a non-empty set $S$ belongs to the coset ring of $H$, then it belongs to the smallest ring of subsets of $H$ containing all cosets of some finite family of open subgroups of $H$. Hence, as $H$ is compact. $S$ is simply a union of cosets of an open subgroup of $H$. Thus the conclusion of Lemma 3.1. could be expressed more explicitly as follows: there are disjoint sets $S_{1}, \ldots, S_{m}$, each a union of cosets of some fixed open subgroup of $H$, with union $H$, and $c_{j} \in \mathrm{T}, a_{j} \in \mathrm{R}^{p}$ and $\gamma_{j} \in \hat{H}$ such that 


$$
g(t, h)=c_{j} \gamma_{j}(h) e^{2 \pi i\left\langle a_{j}, t\right\rangle}, \quad(t, h) \in \bar{W} \times S_{j}, \quad 1 \leqq j \leqq m .
$$

Proof of Theorem 3.1. It is sufficient to prove that if 0 is an interior point in $E$ and $f(0)=1$, then $f$ is equal to a local character in a nbd of 0 . By a well known theorem in structure theory (see for example [8, Theorem 24.30]) the group $G$ is (isomorphic with) the direct product of $\mathrm{R}^{m}$ and a LCA group $G_{0}$ containing a compact open subgroup. By Theorem 2.2. there is then a morphism $\psi: \mathrm{R}^{q} \rightarrow G_{0}$ and open connected nbds $U$ and $V$ of 0 in $\mathrm{R}^{m}$ and $\mathrm{R}^{q}$, respectively, such that the nbd $U \times(\psi(V)+H)$ is contained in the interior of $E$ and the morphism

$$
\sigma:(x, t, h) \rightarrow(x, \psi(t)+h), \quad(x, t, h) \in \mathbf{R}^{m} \times \mathbf{R}^{p} \times H
$$

restricted to $U \times V \times H$ is a local isomorphism. If $V$ is chosen in such a way that $\bar{U} \times(\psi(\bar{V})+H) \subset E$, it follows from Lemma 2.1 that $\left.f \circ \sigma\right|_{\bar{U} \times \bar{V} \times H} \in \mathscr{B}(\bar{U}$ $\times \bar{V} \times H$ ). Hence, by Lemma 3.1 and the remark following it, there is an open nbd $S$ of 0 in $H$ such that $f \circ \sigma$ restricted to $U \times V \times S$ coincides with a character of $R^{m} \times R^{q} \times H$. This proves the theorem.

\section{A stronger local result.}

We will show in this section that in a sense to be made precise below, the number of local characters occurring in Theorem 3.1 is finite. The result will be given in terms of the concept of germs of local characters that we now introduce. If $\Omega$ is the set of all local characters in $G$ the relation "there is a nbd of 0 in $G$ in which $\theta \in \Omega$ and $\theta^{\prime} \in \Omega$ are defined and agree" is an equivalence relation. The germs of local characters will be the equivalence classes of this relation.

THEOREM 4.1. Let $f \in \mathscr{B}(E)$. Then $f$ agrees locally in the interior of $E$ with translates of local characters belonging to at most $p$ different germs times complex numbers of modulus one. Here $p \leqq C=\sup _{n \in \mathrm{Z}_{+}}\left\|f^{n}\right\|_{B(E)}$.

Proof. Let $x_{1}, \ldots, x_{p}$ be any $p$ points in the interior of $E$. By Theorem 3.1 there are a nbd $W$ of 0 in $G$, local characters $\theta_{1}, \ldots, \theta_{p}$ and complex numbers $c_{1}, \ldots, c_{p}$ of modulus one such that

$$
f\left(x+x_{k}\right)=c_{k} \theta_{k}(x), \quad x \in W \quad 1 \leqq k \leqq p .
$$

If we can show that

$$
\theta_{1}, \ldots, \theta_{p} \text { all belong to different germs }
$$

implies that $p \leqq C$, the theorem will follow. Assume (2) and regard $G$ as 
$\mathrm{R}^{m} \times G_{0}$ where $G_{0}$ contains a compact open subgroup. We may suppose that $W$ is so small that for $1 \leqq k \leqq p, \theta_{k}(W) \subset\left\{e^{2 \pi i \tau}|| \tau \mid \leqq \frac{1}{3}\right\}$ and hence

$$
\theta_{k}=1 \text { on each subgroup of } G \text { contained in } W \text {. }
$$

By Theorem 2.2. there is a morphism $\psi: \mathrm{R}^{q} \rightarrow G_{0}$, cubes $\left.Q_{q}=\right]-r, r\left[{ }^{q}\right.$ and $Q_{m}$ $=]-r, r\left[{ }^{m}\right.$ and a compact subgroup $H$ such that the set $Q_{m} \times\left(\psi\left(Q_{q}\right)+H\right)$ is open and contained in $W$. Consider the morphism

$$
\sigma:(y, t, l) \rightarrow(y, \psi(t))+\sum_{k=1}^{p} l_{k} x_{k}, \quad y \in \mathbf{R}^{m}, t \in \mathbf{R}^{q}, l=\left(l_{1}, \ldots, l_{p}\right) \in \mathbf{Z}^{p} .
$$

If $e_{k}$ is the element in $Z^{p}$ with $k$ th coordinate equal to 1 and the others equal to 0 and if $S=\bigcup_{k=1}^{p}\left(Q_{m} \times Q_{q} \times\left\{e_{k}\right\}\right)$, then $\sigma(\bar{S}) \subset E$. Hence, as $\bar{S}$ is compact and $\sigma$ is a morphism, it follows from Lemma 2.1 that the function $g=\left.f \circ \sigma\right|_{\bar{S}}$ satisfies

$$
g \in A(\bar{S}),|g|=1 \text { and }\left\|g^{n}\right\|_{A(\bar{S})} \leqq C, n \in \mathbf{Z}_{+} .
$$

Since the functions $(y, t) \rightarrow \theta_{k}(y, \psi(t)),(y, t) \in Q_{m} \times Q_{q}=Q$ are local characters in $\mathrm{R}^{m+q}$ and thus restrictions to $Q$ of characters (cf. Remark 2 in Section 2) there are $d_{k}=\left(a_{k}, b_{k}\right) \in \hat{\mathrm{R}}^{m+q}$ such that

$$
\theta_{k}(y, \psi(t))=e^{2 \pi i\left(\left\langle y, a_{k}\right\rangle+\left\langle t, b_{k}\right\rangle\right)}, \quad(y, t) \in Q .
$$

Hence, by (1)

$$
g\left(v, e_{k}\right)=c_{k} e^{2 \pi i\left\langle d_{k}, v\right\rangle}, \quad v \in Q, 1 \leqq k \leqq p
$$

By (2) and (3) all $d_{k}$ are different and we will now use a slight modification of an argument due to Y. Domar (see $[6$, p. 4]) to show that this together with (4) and (5) implies $p \leqq C$.

Let $C^{\prime}>C$ be given and choose $\varphi_{n} \in L^{1}\left(\hat{\mathrm{R}}^{s} \times \mathrm{T}^{p}\right),(s=m+q)$, such that $\left\|\varphi_{n}\right\|_{L^{1}}$ $\leqq C^{\prime}$ and $\hat{\varphi}_{n} \mid s=g^{n}$, i.e.

$$
\hat{\varphi}_{n}\left(v, e_{k}\right)=c_{k}^{n} e^{2 \pi i n\left\langle d_{k}, v\right\rangle}, \quad v \in Q, 1 \leqq j \leqq p .
$$

If $\Delta(\xi)=\Delta_{1}\left(\xi_{1}\right) \cdot \ldots \cdot \Delta_{1}\left(\xi_{s}\right), \xi=\left(\xi_{1}, \ldots, \xi_{s}\right) \in \hat{R}^{s}$, where $\Delta_{1}(t)=\max (1-|t|, 0)$, $t \in R$, then $\hat{\Delta} \in L^{1}\left(\mathbf{R}^{s}\right)$ and $\hat{\Delta}=\breve{\Delta}=\Delta$, where $\check{\Delta}(\xi)=\Delta(-\xi)$. For $d \in \hat{R}^{s}, \varepsilon>0$ and $n \in Z_{+}$given we form the function

$$
F_{d, \varepsilon, n}(v, l)=\iint_{\hat{R}^{s} \times T^{p}} \Delta\left(\frac{1}{\varepsilon}\left(\frac{\xi}{n}-d\right)\right) \varphi_{n}(\xi, \eta) e^{-2 \pi i\langle v, \xi\rangle} \cdot e^{-2 \pi i\langle l, \eta\rangle} d \xi d \eta
$$

on $R^{s} \times Z^{p}$, i.e. the Fourier transform of the function

$$
(\xi, \eta) \rightarrow \Delta\left(\frac{1}{\varepsilon}\left(\frac{\xi}{n}-d\right)\right) \varphi_{n}(\xi, \eta), \quad(\xi, \eta) \in \hat{\mathrm{R}}^{s} \times \mathrm{T}^{p} .
$$


By a routine calculation, $(\xi, \eta) \rightarrow \Delta\left(\frac{1}{\varepsilon}\left(\frac{\xi}{n}-d\right)\right),(\xi, \eta) \in \hat{\mathrm{R}}^{s} \times \mathrm{T}^{p}$ is the Fourier transform of the function $h_{\varepsilon n} \in L^{1}\left(R^{s} \times Z^{p}\right)$ given by

$$
h_{\varepsilon n}(u, m)= \begin{cases}(\varepsilon n)^{s} e^{2 \pi i\langle d / \varepsilon, \varepsilon n u\rangle} \hat{\Delta}(\varepsilon n u) & \text { if } m=0 \\ 0 & \text { if } m \neq 0 .\end{cases}
$$

Thus by Parseval's formula

$$
F_{d, \varepsilon, n}(v, l)=\int_{\mathrm{R}^{s}} \sum_{m \in \mathbf{Z}^{p}} h_{\varepsilon n}(u, m) \hat{\varphi}_{n}(u+v, m+l) d u=\int_{\mathrm{R}^{s}} h_{\varepsilon n}(u, 0) \hat{\varphi}_{n}(u+v, l) d u
$$

and hence

$$
F_{d, \varepsilon, n}(v, l)=\int_{\mathrm{R}^{s}} \hat{\varphi}_{n}\left(\frac{u}{\varepsilon n}+v, l\right) e^{2 \pi i\langle d / \varepsilon, u\rangle} \hat{\Delta}(u) d u .
$$

For $n$ large $u / \varepsilon n \in Q$ and then by (5)

$$
\hat{\varphi}_{n}\left(\frac{u}{\varepsilon n}, e_{k}\right) \cdot \hat{\varphi}_{n}\left(0, e_{k}\right)^{-1}=e^{2 \pi i n\left\langle d_{k}, u / \varepsilon n\right\rangle}=e^{2 \pi i\left\langle d_{k} u / \varepsilon\right\rangle},
$$

which shows that the functions $u \rightarrow \hat{\varphi}_{n}\left(u / \varepsilon n, e_{k}\right) \hat{\varphi}_{n}\left(0, e_{k}\right)^{-1}$ converge to the function $u \rightarrow e^{2 \pi i\left\langle d_{k}, u / \varepsilon\right\rangle}$ pointwise in $R^{s}$. By the Lebesgue's dominated convergence theorem, for $1 \leqq k \leqq p$,

$$
\begin{aligned}
& \lim _{n \rightarrow \infty} F_{d, \varepsilon, n}\left(0, e_{k}\right) \cdot \hat{\varphi}_{n}\left(0, e_{k}\right)^{-1} \\
&=\int_{R^{s}} e^{2 \pi i\left\langle d_{k} / \varepsilon, u\right\rangle} \cdot e^{2 \pi i\langle d / \varepsilon, u\rangle} \hat{\Delta}(u) d u=\Delta\left(\frac{d_{k}+d}{\varepsilon}\right) .
\end{aligned}
$$

As $\Delta(0)=1$ and $\left|\hat{\varphi}_{n}\left(0, e_{k}\right)\right|=1$, given $\delta>0$ and $\varepsilon>0$ we can choose $n$ large enough to have

$$
\forall \varepsilon>0\left|F_{-d_{k}, \varepsilon, n}\left(0, e_{k}\right)\right| \geqq 1-\frac{\delta}{p}, \quad 1 \leqq k \leqq p .
$$

With $n$ chosen in this way, by the definition of $F_{d, \varepsilon, n}$,

$$
p-\delta \leqq \sum_{k=1}^{p}\left|F_{-d_{k}, \varepsilon, n}\left(0, e_{k}\right)\right| \leqq \sum_{k=1}^{p} \iint_{R^{3} \times T^{p}} \Delta\left(\frac{1}{\varepsilon}\left(\frac{\xi}{n}+d_{k}\right)\right)\left|\varphi_{n}(\xi, \eta)\right| d \xi d \eta .
$$

But $\Delta\left(1 / \varepsilon\left(\xi / \mathrm{n}+\mathrm{d}_{k}\right)\right)=0$ if not all coordinates of $\xi / n+d_{k}$ have modulus at most $\varepsilon$. As all $d_{k}$ are different, we can choose $\varepsilon$ so small that for each $n$ the functions $\xi \rightarrow \Delta\left(1 / \varepsilon\left(\xi / n+d_{k}\right)\right), \xi \in \mathrm{R}^{s}, 1 \leqq k \leqq p$ have disjoint supports. This implies that

$$
\sum_{k=1}^{p} \iint_{\hat{R}^{s} \times T^{p}} \Delta\left(\frac{1}{\varepsilon}\left(\frac{\xi}{n}+d_{k}\right)\right)\left|\varphi_{n}(\xi, \eta)\right| d \xi d \eta \leqq\left\|\varphi_{n}\right\|_{L^{1}} \leqq C^{\prime} .
$$


Thus $p \leqq C^{\prime}+\delta$ and as $\delta>0$ and $C^{\prime}>C$ was arbitrary, we have proved that $p$ $\leqq C$.

\section{The characterization.}

In this section we will assume that $E$ is compact and the closure of its interior. Let $\mathscr{L}(E)$ be the set of all complex-valued functions on $E$ with the following property: for each $x \in E$ there is a nbd $U$ of 0 in $G$, a local character $\theta: U \rightarrow T$ and $a c \in T$ such that

$$
\forall y \in E \cap(x+U): f(y)=c \theta(y-x) .
$$

We will now prove our main result, i.e. that $\mathscr{B}(E)=\mathscr{L}(E)$, and describe the structure of the functions in $\mathscr{L}(E)$. We start with a simple lemma concerning Diophantine approximation.

Lemma 5.1. Let $0<\varepsilon \leqq \frac{1}{2}$ and $\tau_{1}, \ldots, \tau_{k}$ be real numbers such that

$$
0<\tau_{1}<\frac{\varepsilon}{2} \text { and } 0<\tau_{j}<\frac{\varepsilon}{2} \tau_{j-1}, 2 \leqq j \leqq k .
$$

Then there are infinitely many positive integers $n$ such that the distance from $n \tau_{j}$ to the nearest odd integer is less than $\varepsilon$ for $1 \leqq j \leqq k$.

Proof. Let $h$ be any positive odd integer and $n_{k}$ be the first positive integer for which $n_{k} \tau_{k}>h-\varepsilon$. Then for each integer $l$ with $0 \leqq l \leqq\left[2 \varepsilon / \tau_{k}\right]-2$, we will have $\left|\left(n_{k}+l\right) \tau_{k}-h\right|<\varepsilon$. As the length of the interval $I_{k-1}=\left[n_{k} \tau_{k-1},\left(n_{k}+\left[2 \varepsilon / \tau_{k}\right]\right.\right.$ $\left.-2) \tau_{k-1}\right]$ is at least $\left(\left(2 \varepsilon / \tau_{k}\right)-3\right) \tau_{k-1}>4-3 \tau_{k-1} \geqq \frac{13}{4}$ there is an odd integer, say $h_{k-1}$, such that $\left[h_{k-1}-\varepsilon, h_{k-1}+\varepsilon\right] \subset I_{k-1}$. If $n_{k-1}=n_{k}+l_{k-1}$ is the first integer among the integers $n_{k}+l, 0 \leqq l \leqq\left[2 \varepsilon / \tau_{k}\right]-2$ for which $n_{k-1} \tau_{k-1}>h_{k-1}-\varepsilon$, we will have $\left|\left(n_{k-1}+l\right) \tau_{k-1}-h_{k-1}\right|<\varepsilon$ whenever $0 \leqq l \leqq\left[2 \varepsilon / \tau_{k-1}\right]-2$. Thus the argument can be repeated. Continuing in this way we finally find at least 3 consecutive integers $n$ among the integers $n_{k}+l, 0 \leqq l \leqq\left[2 \varepsilon / \tau_{k}\right]-2$ and odd integers $h_{1}, \ldots, h_{k-1}$ such that

$$
\left|n \tau_{j}-h_{j}\right|<\varepsilon, \quad 1 \leqq j \leqq k-1 .
$$

Since $h$ was arbitrary, the lemma is proved.

LemMA 5.2. If $U$ is an open set in $G$ with $K=\bar{U}$ compact, and if $f \in \mathscr{B}(K)$ and $\left.f\right|_{U}$ is locally constant, then $f(K)$ is finite.

Proof. Suppose that $f(K)$ is infinite. As $\left.f\right|_{U}$ is locally constant, there is a sequence $\left(x_{m}\right)_{m=1}^{\infty}$ in $U$ and a sequence $\left(V_{m}\right)_{m=1}^{\infty}$ of compact symmetric nbds of 0 in $G$ such that 


$$
2 V_{m+1} \subset V_{m}, x_{m}+V_{m} \subset U, f(x)=f\left(x_{m}\right) \text { on } x_{m}+V_{m}
$$

and such that the points $f\left(x_{m}\right) \in \mathrm{T}$ are distinct. Let $H=\bigcap_{m=1}^{\infty} V_{m}$, which is a closed subgroup of $G$. For each open nbd $W$ of 0 in $G$ there is a $k \in Z_{+}$such that $V_{k} \subset W+H$; otherwise, the sequence $V_{k} \backslash(W+H)$ of compact sets has the finite intersection property and hence a non-void intersection, which is impossible. Thus if $\alpha: G \rightarrow G / H$ is the canonical map, the sets $\alpha\left(V_{k}\right)=$ $\left\{x+H \mid x \in V_{k}\right\}, k \in Z_{+}$, form a basis at the origin in $G / H$. Since $K$ is compact, $\alpha(K)$ is compact and therefore the sequence $\left(x_{m}+H\right)_{m=1}^{\infty}$ in $G / H$ has a convergent subsequence. By choosing a subsequence of $\left(x_{m}\right)_{m=1}^{\infty}$ we may if necessary thus assume that $\left(x_{m}+H\right)_{m=1}^{\infty}$ is convergent and

(1) if $c_{m}=f\left(x_{m}\right)$ and $e^{\pi i t_{j}}=\frac{c_{2 j}}{c_{2 j+1}}$, then $0<\tau_{1}<\frac{1}{4}$ and $0<\tau_{j}<\frac{\tau_{j-1}}{4}$, if $j \geqq 2$.

From (1) it follows by Lemma 5.1. that there is an infinite sequence $\left(n_{k}\right)_{k=1}^{\infty}$ of positive integers such that

$$
\left|\left(\frac{c_{2 j}}{c_{2 j+1}}\right)^{n_{k}}-1\right| \geqq \frac{1}{2} \quad \text { if } 1 \leqq j \leqq k .
$$

Since $f \in \mathscr{B}(K)$ there is a bounded sequence $\left(\mu_{k}\right)_{k=1}^{\infty}$ in $M(\Gamma)$ such that $\hat{\mu}_{k}(x)$ $=c_{m}^{n_{k}}$, if $x \in x_{m}+V_{m}$. Now let $\mu$ be a weak*-clusterpoint of $\left(\mu_{k}\right)_{k=1}^{\infty}$. For any finite set of functions $g \in L^{1}(G)$, vanishing outside $x_{m}+V_{m}$, it follows by Parseval's formula that there is a clusterpoint $d_{m}$ of $\left(c_{m}^{n_{k}}\right)_{k=1}^{\infty}$ such that

$$
\int_{G} g(x)\left[\hat{\mu}(x)-d_{m}\right] d x=0 .
$$

As $\hat{\mu}$ is continuous, this implies that $\hat{\mu}(x)=d_{m}$ in the interior of $x_{m}+V_{m}$ and by (2) we have

$$
\left|\frac{d_{2 j}}{d_{2 j+1}}-1\right| \geqq \frac{1}{2}, \quad j \in Z_{+} .
$$

But if $V$ is the interior of the set $\bigcup_{m=2}^{\infty}\left(x_{m}+V_{m+1}\right)$, the function $h: x+H$ $\rightarrow \hat{\mu}(x), x \in V$ is well defined and uniformly continuous on $\alpha(V)$. Since $h\left(x_{m}+H\right)=d_{m}$ and $\left(x_{m}+H\right)_{m=1}^{\infty}$ converges as $m \rightarrow \infty$ we have reached a contradiction.

THEOREM 5.1. If $E$ is compact and the closure of its interior then $\mathscr{B}(E)=\mathscr{L}(E)$. 
Proof. Suppose that $f \in \mathscr{B}(E)$ and let $U$ be the interior of $E$. By Theorem 4.1 there are $p$ local characters $\theta_{k}: W_{0} \rightarrow$ T belonging to different germs such that the open sets

$$
\begin{gathered}
U_{k}=\left\{x \in U \mid \exists \operatorname{nbd} W \text { of } 0 \text { in } G \text { such that } W \subset W_{0}, x+W \subset U\right. \text { and } \\
\left.\forall y \in x+W, f(y)=f(x) \theta_{k}(y-x)\right\}
\end{gathered}
$$

form a partition of $U$.

Let $x_{0} \in \bar{U}_{k}$ and let $V_{0}$ be an open nbd of 0 in $G$ with $\bar{V}_{0}$ compact and contained in $W_{0}$. The function

$$
x \rightarrow \frac{f(x)}{\theta_{k}\left(x-x_{0}\right)}, \quad x \in \overline{\left(x_{0}+V_{0}\right) \cap U_{k}},
$$

is in $\mathscr{B}\left(\overline{\left.\left(x_{0}+V_{0}\right) \cap U_{k}\right)}\right.$ and is locally constant in $\left(x_{0}+V_{0}\right) \cap U_{k}$. Hence, by Lemma 5.2, it has a finite range and this implies that the function is constant in $\bar{U}_{k} \cap\left(x_{0}+V_{k}\right)$, for some nbd $V_{k}$ of 0 in $G$. If $x_{0}$ also belongs to $\bar{U}_{l}$ for some $l \neq k$, there is an open nbd $V$ of 0 in $G$ with $\bar{V}$ compact such that if $Q_{k}=\left(x_{0}+V\right) \cap U_{k}$ and $Q_{l}=\left(x_{0}+V\right) \cap U_{l}$, then there are $c_{k}$ and $c_{l}$ in $\mathrm{T}$ such that

$$
f(x)= \begin{cases}c_{k} \theta_{k}\left(x-x_{0}\right) & \text { if } x \in \bar{Q}_{k} \\ c_{l} \theta_{l}\left(x-x_{0}\right) & \text { if } x \in \bar{Q}_{l} .\end{cases}
$$

Thus, if $\theta=\theta_{k} / \theta_{l}$ and $c=c_{k} / c_{l}$, the function $g$ defined on $\bar{Q}_{k} \cup \bar{Q}_{l}$ by

$$
g(x)= \begin{cases}1 & \text { if } x \in \bar{Q}_{k} \\ c \theta\left(x-x_{0}\right) & \text { if } x \in \bar{Q}_{l}\end{cases}
$$

is in $\mathscr{B}\left(\bar{Q}_{k} \cup \bar{Q}_{l}\right)$. Hence there is a sequence $\left(\mu_{n}\right)_{n=1}^{\infty}$ in $M(\Gamma)$ such that $\hat{\mu}_{n}(x)$ $=g^{n}(x)$ if $x \in \bar{Q}_{k} \cup \bar{Q}_{l}$ and with $C=\sup _{n \in Z_{+}}\left\|\mu_{n}\right\|_{M(\Gamma)}<\infty$. With $\mu_{0}=\delta_{0}$, the Dirac measure at 0 , let $\lambda_{n}=2^{-n} \sum_{k=0}^{n}\left(\begin{array}{l}n \\ k\end{array}\right) \mu_{k}$, a sequence in $M(\Gamma)$ bounded by $C$ and such that

$$
\hat{\lambda}_{n}(x)= \begin{cases}1 & \text { if } x \in \bar{Q}_{k} \\ \left(\frac{1+c \theta\left(x-x_{0}\right)}{2}\right)^{n} & \text { if } x \in \bar{Q}_{l} .\end{cases}
$$

Since $\theta_{k}$ and $\theta_{l}$ belong to different germs, the set

$$
F=\left\{x \in Q_{k} \mid c \theta\left(x-x_{0}\right)=1\right\}
$$

is of Haar measure 0 , and

$$
\lim _{n \rightarrow \infty} \hat{i}_{n}(x)= \begin{cases}1 & \text { if } x \in Q_{k} \cup F \\ 0 & \text { if } x \in Q_{l} \backslash F\end{cases}
$$


Now let $\lambda \in M(\Gamma)$ be a weak*-clusterpoint of $\left(\hat{\lambda}_{n}\right)_{n=1}^{\infty}$. It is easy to see that

$$
W=\left\{x \in Q_{k} \cup Q_{l} \mid \hat{\lambda}(x) \neq \lim _{n \rightarrow \infty} \hat{\lambda}_{n}(x)\right\}
$$

is of Haar measure 0 . Hence $\hat{i}(x)=0$ or 1 for each $x \in Q_{k} \cup Q_{l}$ by continuity. Thus

$$
\begin{aligned}
&\left\{x \in Q_{l} \mid\right.\hat{i}(x) \neq 0\}=\left\{x \in Q_{l} \mid \hat{i}(x)=1\right\} \\
&=\{x \in F \mid \hat{i}(x)=1\} \cup\left\{x \in Q_{l} \backslash F \mid \hat{\hat{\lambda}}(x)=0\right\} \subset F \cup W
\end{aligned}
$$

which shows that $\hat{\hat{\lambda}}(x)=0$ on $Q_{l}$. In the same way it is shown that $\hat{\lambda}(x)=1$ on $Q_{k}$ and the continuity of $\hat{i}$ implies that $\bar{Q}_{k} \cap \bar{Q}_{l}=\varnothing$. This shows that if $x_{0} \in \bar{U}_{k}$, then $x_{0} \notin \bar{U}_{l}$ for any $l \neq k$. Thus $\mathscr{B}(E) \subset \mathscr{L}(E)$. Since $E$ is compact we have $\mathscr{L}(E) \subset \mathscr{B}(E)$ (cf. the introduction).

Remark. The proof actually shows that if $E$ is the closure of its interior (compact or not) and if $f \in \mathscr{B}(E)$ then $f$ agrees locally on $E$ with translates of $p$ $\leqq \sup _{n \in Z_{+}}\left\|f^{n}\right\|_{B(E)}$ local characters belonging to different germs times constants of modulus one. However, a function with such a representation need not even belong to $B(E)$ if $E$ is non-compact. As an example. the function $f$ defined by

$$
f(x)=\left\{\begin{aligned}
-1 & \text { if } x \leqq 0 \\
1 & \text { if } x \geqq 1
\end{aligned}\right.
$$

does not belong $B(]-\infty, 0] \cup[1, \infty[)$. If it did, there would be a measure $\mu \in M(\mathrm{R})$ with $\hat{\mu}(x)=0$ if $x \leqq 0$ and $\hat{\mu}(x)=1$ if $x \geqq 1$. Thus the measure $v=\mu+\delta_{0}$ satisfies $\hat{v}(x)=0$ for $x \leqq 0$ and $\hat{v}(x)=2$ for $x \geqq 1$. But by the theorem of $\mathrm{F}$. and M. Riesz (see for example [12, Theorem 8.2.7]), a $v \in M(R)$ with $\hat{v}(x)=0$ if $x \leqq 0$ is absolutely continuous. Hence by the Riemann-Lebesgue lemma $\lim _{x \rightarrow+\infty} \hat{v}(x)=0$, a contradiction.

The rest of this section will be devoted to an explicit description of the functions in $\mathscr{L}(E)$ for a set $E$ which is compact and which is the closure of its interior. We start with a definition.

Definition 5.1. If $U$ is an open nbd of 0 in a LCA group $G$, a function $\omega: U$ $\rightarrow \mathrm{T}$ is said to be a local quasi-character if there are open nbds $V \subset W \subset U$ of 0 in $G$ such that

$$
\left.\omega\right|_{W} \text { is a local character }
$$

$$
\forall x \in U \exists x_{1}, \ldots, x_{m} \in W \text { such that } x=\sum_{i=1}^{m} x_{i} \text { and } \omega(x)=\prod_{i=1}^{m} \omega\left(x_{i}\right)
$$




$$
\forall x \in U \forall x^{\prime} \in V: x+x^{\prime} \in U \Rightarrow \omega\left(x+x^{\prime}\right)=\omega(x) \omega\left(x^{\prime}\right) .
$$

The next simple lemma shows that the class of local quasi-characters coincides with that of functions in $G$, induced of characters of groups, locally isomorphic with $G$. More precisely:

Lemma 5.3. The function $\omega: U \rightarrow T$ is a local quasi-character in $G$ if and only if there is a LCA group $G^{\prime}$, an open morphism $\alpha: G^{\prime} \rightarrow G a \beta \in \hat{G}^{\prime}$ and open nbds $V^{\prime} \subset W^{\prime} \subset U^{\prime}$ of 0 in $G^{\prime}$ such that

$$
\left.\alpha\right|_{W^{\prime}}: W^{\prime} \rightarrow \alpha\left(W^{\prime}\right) \text { is a local isomorphism of } G^{\prime} \text { with } G .
$$

$U^{\prime}$ is contained in the subgroup of $G^{\prime}$ generated by $W^{\prime}$.

$$
\alpha \text { is injective on } U^{\prime}+V^{\prime}, \alpha\left(U^{\prime}\right)=U \text { and } \omega=\beta \circ\left(\left.\alpha\right|_{U^{\prime}}\right)^{-1} \text {. }
$$

Proof. Suppose that $\omega: U \rightarrow T$ is a local quasi-character. We introduce the group $\hat{G}$ and the maps $\alpha$ and $\beta$ constructed from $G$ and the local character $\left.\omega\right|_{W}$ in Theorem 2.1. If $\tilde{U}$ is the graph of $\omega$, then $\tilde{U} \subset \bar{G}$ by (2) in Definition 5.1. From (3) it follows that $\hat{U}$ is open in $\widehat{G}$, that $\alpha(\hat{U})=U$ and that $\omega=\beta \circ\left(\left.\alpha\right|_{\hat{U}}\right)^{-1}$. Furthermore, (3) implies that if $\hat{V}$ is the graph of $\left.\omega\right|_{V}$, then $(\hat{U}-\hat{U}+\hat{V}) \cap \operatorname{Ker} \alpha$ $=\{0\}$. Hence $\alpha$ is injective on $\tilde{U}+\hat{V}_{1}$, for each open nbd $\hat{V}_{1}$ of 0 in $\hat{G}$ that satisfies $\hat{V}_{1}-\hat{V}_{1} \subset \hat{V}$. This proves the necessity. The sufficiency is established by a straightforward inspection.

It is readily seen that a local quasi-character is a uniformly continuous function. If $\omega: U \rightarrow \mathrm{T}$ is a local quasi-character and $\bar{U}$ is compact, then the continuous extension of $\omega$ to $\bar{U}$ is in $\mathscr{B}(\bar{U})$ by Theorem 2.3 and the preceding lemma. However, if $\bar{U}$ is non-compact, $\omega$ will in general not even belong to $B(\bar{U})$. We will give an example of this in the next section where a main point is to seek conditions, less restrictive than compactness of $\bar{U}$, which imply that $\omega \in \mathscr{B}(\bar{U})$.

THEOREM 5.2. Suppose that $E$ is a compact set which is the closure of its interior. Then $f \in \mathscr{L}(E)$ if and only if there is a partition of $E$ into disjoint compact sets $E_{1}, \ldots, E_{m}$, each $E_{i}$ contained in an open set $U_{i}, x_{i} \in E$ and local quasi-characters $\omega_{i}: U_{i}-x_{i} \rightarrow \mathrm{T}$ and $c_{i} \in \mathrm{T}, 1 \leqq i \leqq m$, such that

$$
f(x)=c_{i} \omega_{i}\left(x-x_{i}\right), \quad x \in E_{i} .
$$

Proof. As it is immediately seen that a function with the indicated representation is in $\mathscr{L}(E)$ it is enough to show the converse statement. Suppose that $f \in \mathscr{L}(E)$. From the definition of $\mathscr{L}(E)$ it follows, by compactness, that 
there are $x_{1}, \ldots, x_{n} \in E$, open nbds $V_{1}, \ldots, V_{n}$ of 0 in $G$, local characters $\theta_{i}: V_{i}$ $\rightarrow \mathrm{T}$ and $c_{i} \in \mathrm{T}$ such that

$$
E \subset \bigcup_{i=1}^{n}\left(x_{i}+V_{i}\right)
$$

and

$$
f(x)=c_{i} \theta_{i}\left(x-x_{i}\right) \text { if } x \in E \cap\left(x_{i}+V_{i}\right) .
$$

Let $Q$ be the interior of $E$ and $Q_{i}=Q \cap\left(x_{i}+V_{i}\right)$. Assume that $\bar{Q}_{i} \cap \bar{Q}_{j} \neq \varnothing$ and choose a $x_{0} \in \bar{Q}_{i} \cap \bar{Q}_{j}$. As $x_{0} \in E$ we have $x_{0} \in x_{k}+V_{k}$ for some $k$ with $1 \leqq k \leqq n$ by (1) and since $x_{k}+V_{k}$ is a nbd of $x_{0}$, we have

$$
\left(x_{k}+V_{k}\right) \cap Q \cap\left(x_{i}+V_{i}\right) \neq \varnothing,\left(x_{k}+V_{k}\right) \cap Q \cap\left(x_{j}+V_{j}\right) \neq \varnothing .
$$

Since these intersections are open, (2) implies that $\theta_{i}$ and $\theta_{j}$ belong to the same germ. Hence, if $\boldsymbol{g}_{1}, \ldots, \boldsymbol{g}_{p}$ are all the different germs to which the local characters $\theta_{i}(1 \leqq i \leqq n)$ belong and $M_{j}=\left\{i \mid \theta_{i} \in g_{j}\right\}$, then $E$ is decomposed into the disjoint compact sets $E_{j}=U_{i \in M}, \bar{Q}_{i}, 1 \leqq j \leqq p$. If $x \in E_{j}$ then $x \in \bar{Q}_{i}$ $=\overline{\left[Q \cap\left(x_{i}+V_{i}\right)\right]}$ for some $i \in M_{j}$ and by (1), there is a $k$ such that $x \in x_{k}+V_{k}$. As $x_{k}+V_{k}$ is a nbd of $x$ we have $\left(x_{k}+V_{k}\right) \cap Q \cap\left(x_{i}+V_{i}\right) \neq \varnothing$, which implies that $\theta_{i}$ and $\theta_{k}$ are in the same germ. Thus $k \in M_{j}$ and therefore

$$
E_{j} \subset \bigcup_{i \in M_{j}}\left(x_{i}+V_{i}\right) \text {. }
$$

By using a refinement of the cover $\left(x_{i}+V_{i}\right)$ of $E_{j}$ if necessary, we may assume that there is an open nbd $W_{j}$ of 0 in $G$ and a local character $x_{j}: W_{j} \rightarrow \mathrm{T}$ such that $\bigcup_{i \in M_{,}} \bar{V}_{i} \subset W_{j}$ and $\theta_{i}=x_{j}$ on $V_{i}, i \in M_{j}$.

Now let $G_{j}$ be the open subgroup of $G$ generated by $W_{j}$. If $K_{j 1}, \ldots, K_{j q}$ are all the different cosets of $G_{j}$ to which $x_{i}, i \in M_{j}$, belongs and $M_{j k}$ $=\left\{i \in M_{j} \mid x_{i} \in K_{j k}\right\}$, then $E_{j}$ is decomposed into the disjoint compact sets $E_{j k}=E_{j} \cap K_{j k}, 1 \leqq k \leqq q$. Since

$$
E_{j k}=\bigcup_{i \in M_{j k}} \bar{Q}_{i} \subset \bigcup_{i \in M_{j k}}\left(x_{i}+V_{i}\right),
$$

to finish the proof, it is sufficient to show that if $M \subset M_{j k}$ and $f$ coincides with a local quasi-character on the compact nbd $F=\bigcup_{i \in M} \bar{Q}_{i}$ of 0 in $G$ and $F$ meets $\bar{Q}_{l}$, $l \in M_{j k}$, then $f$ coincides with a local quasi-character on $F \cup \bar{Q}_{l}$.

Let $\tilde{G}_{j}$ be the group and $\alpha_{j}$ and $\beta_{j}$ be the maps constructed from the local character $\theta_{j}: W_{j} \rightarrow \mathrm{T}$, as in Theorem 2.1. If $\hat{F}$ and $\hat{N}$ are the graphs of $\left.f\right|_{F}$ and $\left.f\right|_{\Phi_{1}}$ respectively, the hypothesis implies that $\tilde{F}$ and $\tilde{N}$ are compact sets in $\tilde{G}$ and

$$
f \circ \alpha_{j}(\tilde{x})= \begin{cases}\beta_{j}(\tilde{x}) & \text { if } \tilde{x} \in \tilde{F} \\ C_{l} \beta_{j}(\hat{x}) & \text { if } \tilde{x} \in \tilde{N}\end{cases}
$$


for some constant $C_{l} \in \mathrm{T}$. By the definition of $\alpha_{j}$ and $\beta_{j}$,

$$
\operatorname{Ker} \alpha_{j} \cap \operatorname{Ker} \beta_{j}=\{0\} .
$$

Since $\alpha_{j}(\tilde{F}) \cap \alpha_{j}(\tilde{N})=F \cap \bar{Q}_{l} \neq \varnothing$, by (3) there is a unique $\tilde{h} \in \operatorname{Ker} \alpha$ such that $\tilde{h}+\tilde{N}$ meets $\tilde{F}$. As this $\tilde{h}$ satisfies $\beta_{j}(\tilde{h})=C_{l}$, it follows that

$$
f \circ \alpha_{j}(\hat{x})=\beta_{j}(\hat{x}) \quad \text { if } \hat{x} \in \tilde{F} \cup(\hat{h}+\hat{N}) .
$$

By (3) it follows from (4) that $\alpha_{j}$ is injective on $\tilde{F} \cup(\hat{h}+\hat{N})$. Since this set is compact, there is a nbd $\hat{V}$ of 0 in $\widehat{G}$ such that $\alpha_{j}$ is injective on $[\hat{F} \cup(\hat{h}+\hat{N})]+\hat{V}$. If $\hat{V}_{1}$ is a nbd of 0 in $\hat{G}$ with $2 \hat{V}_{1} \subset \hat{V}$ and $\hat{U}=\hat{F}+(\hat{h}+\hat{N})+\hat{V}_{1}$ then Lemma 5.3. implies that $\beta_{j} \circ\left(\alpha_{j} \mid \tilde{U}\right)^{-1}$ is a local quasi-character on $\alpha_{j}(\tilde{U})$, which by (4) coincides with $f$ on $F \cup \bar{Q}_{l}$.

\section{Some results for non-compact sets.}

In all of this section it will be assume that $E$ is the closure of its interior and, in general, is not compact. We first show that additional conditions on $E$ are needed to have a representation of the functions in $\mathscr{B}(E)$ resembling that in Theorem 5.2., with a finite number of constants. One sufficient condition is that the interior of $E$ is connected. In that case we show that a functions in $\mathscr{B}(E)$ coincides on $E$ with a translate of a local quasi-character times a complex number of modulus one.

However, local quasi-characters are in general not even restrictions of Fourier-Stieltjes transforms. We end this section with a discussion of the conditions on a closed nbd $E$ of 0 in $G$, under which any local quasi-character defined on a nbd of $E$ belongs to $\mathscr{B}(E)$. We begin with some examples.

Example 6.1. Let $G$ be a discrete group and $E$ a Sidon set in $G$ (see [12, p. 121]). Then there is a constant $C$ such that

$$
f \in L^{\infty}(E) \Rightarrow f \in B(E) \text { and }\|f\|_{B(E)} \leqq C\|f\|_{L^{\alpha}(E)} .
$$

Thus any $f \in L^{\infty}(E)$ with $|f|=1$ on $E$ is in $\mathscr{B}(E)$.

The next two examples depend on properties of certain discrete subsets in LCA groups, studied in detail in [9]. A subset $L$ of a LCA group $G$ is a coherent set of frequencies if there exists a $C>0$ and a compact set $K$ in $\Gamma=\hat{G}$ such that for each trigonometric polynomial $P(\gamma)$ with frequencies in $L$ we have

$$
\sup _{\gamma \in \Gamma}|P(\gamma)| \leqq C \sup _{\gamma \in K}|P(\gamma)| .
$$

When this holds, the pair $(K, C)$ is said to be suitable for $L$. If $\operatorname{Hom}\left(L_{d}, T\right)$ denotes the group of restrictions to $L$ of all homomorphisms of the group $G$ 
with the discrete topology into $\mathrm{T}$, an equivalent condition is that

$$
\operatorname{Hom}\left(L_{d}, T\right) \subset B(L) .
$$

The following result, due to Y. Meyer, is the reason for our interest in coherent sets of frequencies. We refer to $[9$, p. 126] for the proof.

THEOREM 6.1. Let $L$ be a coherent set of frequencies in $G$ and assume that $\Gamma=\hat{G}$ is metrizable and separable. Then there is a compact nbd $V$ of 0 in $G$ such that the sets $l+V, l \in L$, are pairwise disjoint and such that if $H$ is the homeomorphism of $L+V$ onto $L \times V$ given by $H(l+t)=(l, t), l \in L, t \in V$, there is $C>0$ such that,

$$
g \in B(L \times V) \Rightarrow g \circ H \in B(L+V) \text { and }\|g \circ H\|_{B(L+V)} \leqq C\|g\|_{B(L \times V)} .
$$

EXAMPLE 6.2. Let $G$ be a LCA group, with $\Gamma$ separable and metrizable, and let $L$ be a coherent set of frequencies in $G$. By Theorem 6.1 there is a compact nbd $V$ of 0 in $G$ such that for any $b \in \operatorname{Hom}\left(L_{d}, \mathrm{~T}\right)$ the function $f$ defined on $E$ $=L+V$ by

$$
f(x)=b(l) \quad \text { if } x \in l+V, l \in L,
$$

is in $\mathscr{B}(E)$.

A set $S$ of real numbers is a topological Sidon set if each $f \in L^{\alpha}(S)$ is the restriction to $S$ of the Fourier-Stieltjes transform of a $\mu \in M(\mathrm{R})$ (see $[9, \mathrm{p}$. 183]). As an example, the set $L=\left\{a^{n} \mid n \in Z_{+}\right\}$where $a \in \mathbb{R}, a>1$, is a topological Sidon set (see [9, Theorem II]). We finish our set of examples of functions in $\mathscr{B}(E)$ with those immediately derived from the following result, borrowed from [9, Theorem VI, p. 192].

THEOREM 6.2. Let $L$ be a topological Sidon set of real numbers and $d_{L}$ the infimum of the distances between two distinct point of $L$. For each $d$ in $] 0, d_{L} / 2[$ there is a constant $C(d)$, which is an increasing function of $d$, such that any bounded function $b: L+[-d, d] \rightarrow C$ which is constant on each interval $[l-d$, $l+d]$, is the restriction to $L+[-d, d]$ of the Fourier transform of a measure with norm not exceeding $C(d) \sup _{l \in L}|b(l)|$.

We will now assume that $U$, the interior of $E$, is connected. Without loss of generality we may also assume that $U$ is a nbd of 0 in $G$, that $f(0)=1$ and that the group $G$ is connected. It is then of the form $R^{m} \times K$ where $m \geqq 0$ and $K$ is a compact connected group (cf. for example [8, Theorem 9.8]).

THEOREM 6.3. Let $U$ be an open connected nbd of 0 in the connected LCA 
group $G$ and let $E=\bar{U}$. Suppose that $f \in \mathscr{B}(E)$ and $f(0)=1$. Then there are a connected LCA group $G^{\prime}$, an epimorphism $\alpha: G^{\prime} \rightarrow G, a \beta \in \hat{G}^{\prime}$ and an open connected nbd $U^{\prime}$ of 0 in $G^{\prime}$ such that with $E^{\prime}=\bar{U}^{\prime}$.

(1) there is a $g \in B\left(G^{\prime}\right)$ with $g=1$ on $E^{\prime}$ and $g=0$ on $E^{\prime}+h$ for each $h \in \operatorname{Ker} \alpha$, $h \neq 0$,

(2) $\operatorname{Ker} \alpha \cap \operatorname{Ker} \beta=\{0\}$.

(3) $E=\alpha\left(E^{\prime}\right)$ and $f=\beta \circ\left(\left.\alpha\right|_{E^{\prime}}\right)^{-1}$.

Remark. Since $g$ is uniformly continuous, (1) implies that there is a nbd $W^{\prime}$ of 0 in $G^{\prime}$ such that $\alpha$ is injective on $E^{\prime}+W^{\prime}$. Hence, by Lemma 5.3, the theorem shows in particular that if $f \in \mathscr{B}(E)$, then $f$ coincides on $E$ with a local quasicharacter.

Proof. As $U$ is connected there is, by Theorem 4.1., a local character $\theta: W_{0}$ $\rightarrow$ T such that for each $x \in U$, there is a nbd $W_{x}$ of 0 in $G$ with $W_{x} \subset W_{0}, x+W_{x}$ $\subset U$ and such that $f(x+y)=f(x) \theta(y)$ for all $y \in W_{x}$. Let $U_{1}$ be the set of all $x \in U$ for which there are $x_{1}, \ldots, x_{k} \in W_{0}$ such that $x=\sum_{j=1}^{k} x_{i}$ and $f(x)$ $=\prod_{i=1}^{k} \theta\left(x_{i}\right)$. Of course, $k$ may vary. Then $U_{1} \supset W_{0}$ and if $x \in U_{1}$ and $W_{x}$ is a nbd of 0 such that $W_{x} \subset W_{0}, x+W_{x} \subset U$ and $f^{\prime}(y)=f(x) \theta(y)$ for all $y \in W_{x}$, then trivially $x+W_{x} \subset U_{1}$. Hence $U_{1}$ is a non empty open subset of $U$. As it is equally simple to show that $U_{1}$ is closed in $U$, the connectedness of $U$ implies that $U_{1}=U$.

Let $\tilde{G}$ be the group and $\alpha$ and $\beta$ be the maps constructed from the local character $\theta: W_{0} \rightarrow \mathrm{T}$ as in Theorem 2.1. and let $\tilde{U}$ be the graph of $\left.f\right|_{U}$. By what has been shown above, $\hat{U} \subset \bar{G}, \alpha(\tilde{U})=U$ and $f \circ \alpha=\beta$ on $\tilde{U}$. Since $\operatorname{Ker} \alpha \cap \operatorname{Ker} \beta$ $=\{0\}$, which proves (2), this implies that $\left.\alpha\right|_{\tilde{U}}$ is injective and thus $\left.\alpha\right|_{\tilde{U}}: \tilde{U} \rightarrow U$ is a homeomorphism. Hence $\tilde{U}$ is connected and as $\tilde{G}$ is generated by $\tilde{U}$, it is also connected.

We will now prove (1) from which (3) easily follows. Since $f \in \mathscr{B}(E)$, the function $f \circ \alpha$ is in $\mathscr{B}\left(\alpha^{-1}(E)\right)$ by Lemma 2.1. and as $\beta$ is a character, $(f \circ \alpha / \beta) \in \mathscr{B}\left(\alpha^{-1}(E)\right)$. Hence as

$$
\bigcup_{h \in \operatorname{Ker} \alpha}(\tilde{U}+\tilde{h})=\alpha^{-1}(U) \subset \alpha^{-1}(E),
$$

there is a $C>0$ and a sequence $\mu_{n} \in M(\hat{\Gamma})$, where $\tilde{G}$ is the dual group of $\hat{\Gamma}$, such that,

$$
\hat{\mu}_{n}(\hat{x})=\beta(\hat{h})^{-n} \quad \text { if } \hat{x} \in \hat{U}+\hat{h}
$$

and

$$
\sup _{n \in \mathbf{Z}_{+}}\left\|\mu_{n}\right\|_{M(\tilde{\Gamma})} \leqq C .
$$


If $\mu_{0}=\delta_{0}$ and if

$$
v_{n}=2^{-n} \sum_{k=0}^{n}\left(\begin{array}{l}
n \\
k
\end{array}\right) \mu_{k}
$$

then $\left\|v_{n}\right\|_{M(\tilde{T})} \leqq C$ and for each $\tilde{x} \in \hat{U}+\hat{h}$

$$
\hat{v}_{n}(\tilde{x})=2^{-n} \sum_{k=0}^{n}\left(\begin{array}{l}
n \\
k
\end{array}\right) \beta(\tilde{h})^{-k}=\left(\frac{1+\beta(\hat{h})^{-1}}{2}\right)^{n} .
$$

Since $\operatorname{Ker} \alpha \cap \operatorname{Ker} \beta=\{0\}$ it follows that

$$
\lim _{n \rightarrow \infty} \hat{v}_{n}(\hat{x})= \begin{cases}1 & \text { if } \hat{x} \in \hat{U} \\ 0 & \text { if } \hat{x} \in \hat{U}+\hat{h}, \hat{h} \in \operatorname{Ker} \alpha, \hat{h} \neq 0 .\end{cases}
$$

Let $v$ be a weak ${ }^{*}$-clusterpoint of $\left(v_{n}\right)_{n=1}^{\infty}$. As $\hat{v}$ is continuous and $\hat{U}+\hat{h}$, $\tilde{h} \in \operatorname{Ker} \alpha$, is open it follows (cf. the proof of Lemma 5.2) that

$$
\hat{v}(\hat{x})= \begin{cases}1 & \text { if } \hat{x} \in \overline{\tilde{U}} \\ 0 & \text { if } \hat{x} \in \bar{U}+\hat{h}, \hat{h} \in \operatorname{Ker} \alpha, \hat{h} \neq 0 .\end{cases}
$$

EXAMPLE 6.3. Let $G=R \times T$ and $\alpha: R^{2} \rightarrow R \times T$ be the map given by

$$
\alpha(x, y)=(x, y+Z), \quad(x, y) \in \mathbf{R}^{2} .
$$

Furthermore, let $g: R \rightarrow R$ be the function defined by

$$
g(x)= \begin{cases}0 & \text { if } x \leqq 0 \\ b x & \text { if } x>0, b \in \mathbf{R}_{+},\end{cases}
$$

and let $\beta \in \hat{\mathrm{R}}^{2}$ be defined by $\beta(x, y)=e^{2 \pi i a y},(x, y) \in \mathrm{R}^{2}$, where $a$ is irrational. If

$$
U^{\prime}=\left\{(x, y) \in \mathbf{R}^{2}|| y-g(x) \mid<\delta\right\}
$$

and $\delta>0$ is sufficiently small, then there is a nbd $V^{\prime}$ of 0 in $R^{2}$ such that $\alpha$ is injective on $U^{\prime}+V^{\prime}$.

In this case, the function $f=\beta \circ\left(\left.\alpha\right|_{U^{\prime}}\right)^{-1}$ is a local quasi-character, defined on $U=\alpha\left(U^{\prime}\right)$. However $f \notin B(\bar{U})$. If $f \in B(\bar{U})$, then $f \circ \alpha \in B\left(\alpha^{-1}(\bar{U})\right)$ by Lemma 2.1 and hence, by the theorem above, the function which is 1 on $\bar{U}^{\prime}$ and 0 on $\bar{U}^{\prime}$ $+(0, k), k \in \mathbf{Z}, k \neq 0$, would belong to $B\left(\bar{U}^{\prime}+(\{0\} \times \mathbf{Z})\right)$. Since $\bar{U}^{\prime}+(\{0\} \times \mathbf{Z})$ contains the set

$$
\left\{(x, 0) \in \mathbf{R}^{2} \mid x \leqq 0 \text { or } x=\frac{k}{b}, k \in \mathbf{Z}_{+}\right\},
$$

this implies that the function which is 1 if $x \leqq 0$ and is 0 if $x=k / b, k \in \mathbf{Z}_{+}$, would belong to $\left.B(]-\infty, 0] \cup b^{-1} Z_{+}\right)$. If it did, we could find a $\mu \in M(R)$ such that $\hat{\mu}(x)=1$ if $x \leqq 0, \mu(x)=0$ if $x=k / b, k \in Z_{+}$. Then the measure $v=\mu-\delta_{0}$ 
satisfies $\hat{v}(x)=0$ if $x \leqq 0, \hat{v}(x)=-1$ if $x=k / b, k \in \mathbf{Z}_{+}$, which contradicts the $\mathrm{F}$. and M. Riesz theorem (cf. the remark following Theorem 5.1).

If the conditions (1), (2) and (3) of Theorem 6.3 are satisfied, does the function $f=\beta \circ\left(\left.\alpha\right|_{U^{\prime}}\right)^{-1}$ then belong to $B\left(\overline{\alpha(U)^{\prime}}\right)$ ? As a corollary to the theorem below, we show that in a special case, these conditions are also sufficient. For the theorem, we need some new notations.

Let $G$ be a LCA group with dual group $\Gamma$ and let $H$ be a discrete subgroup of $G$, isomorphic with some subgroup of $\mathrm{T}_{d}$ and with annihilator $\Lambda$ in $\Gamma$. The Haar measure $d h$ of $H$ will be normalized so that each point of $H$ has mass one. The Haar measure $d \hat{\lambda}$ of $\Lambda$ is chosen in such a way that Poisson's formula holds (cf. for example [11, 5.1 p. 120]).

If $V$ is an open subset in $G$ we denote by $S(V)$ the subspace of $L^{1}(\Gamma)$ consisting of all continuous functions $\varphi$ such that supp $\hat{\varphi}$, the support of $\hat{\varphi}$, is a compact subset of $V$. We simply write $S$ for $S(G)$.

THeOREM 6.4. Let $U$ be an open nbd of 0 in $G$ and $\alpha: G \rightarrow G / H$ be the canonical map. Then the following conditions are equivalent

(a) there is a $\beta \in \Gamma$ with $\left.\beta\right|_{H}$ injective for which $\beta \circ\left(\left.\alpha\right|_{\bar{U}}\right)^{-1} \in B(\overline{\alpha(U)})$ and $\sup _{n \in Z_{+}}\left\|\left(\beta \circ\left(\left.\alpha\right|_{\bar{U}}\right)^{-1}\right)^{n}\right\|_{B(\alpha(U))} \leqq C$.

(b) there exists a $v \in M(\Gamma)$ such that $\hat{v}(x)=1$ if $x \in \bar{U}$ and $\hat{v}(x)=0$ if $x \in$ $\bar{U}+h, h \in H, h \neq 0$, and such that for each $\varphi \in S(U+H)$ and $\psi \in S$

$$
\left|\int_{\Lambda} \varphi(-\lambda)(\psi * v)(\lambda) d \hat{\lambda}\right| \leqq C\|\varphi\|_{L^{a}(\Gamma)}\|\psi\|_{L^{1}(\Gamma)} .
$$

(c) for each $f \in B(\bar{U})$ we have $f_{\circ}\left(\left.\alpha\right|_{\bar{U}}\right)^{-1} \in B(\overline{\alpha(U)})$ and-

$$
\left\|f \circ\left(\left.\alpha\right|_{\bar{U}}\right)^{-1}\right\|_{B(\overline{\alpha(U)})} \leqq C\|f\|_{B(\bar{U})} .
$$

It is convenient to preceed the proof with a lemma, enabling us to work in $G$ rather than in $G / H$. For a proof, see for example [7, Theorem 5.3].

LemMa 6.1. Let $H$ be a closed subgroup of $G$ and $\alpha: G \rightarrow G / H$ be the canonical map. Suppose that $V$ is an open set in $G / H$, that $E=\bar{V}$ and that $f \in C(E)$. Then $f \in B(E)$ if and only if $f \circ \alpha \in B\left(\alpha^{-1}(E)\right)$, and in this case $\|f\|_{B(E)}=\|f \circ \alpha\|_{B\left(\alpha^{-1}(E)\right) \text {. }}$

We will also use the following result, essentially an easy consequence of the Hahn-Banach theorem (see for example [9, Proposition 8]).

Lemma 6.2. Let $V$ be an open subset in $G$ and $f$ be a continuous function on $E=\bar{V}$. If there is a constant $C$ such that $\left|\int_{G} f(x) \hat{\varphi}(x) d x\right| \leqq C\|\varphi\|_{\infty}$ for each $\varphi \in S(V)$, then $f \in B(E)$ and $\|f\|_{B(E)} \leqq C$. 
Proof of THE THEOREM. Since $H$ is isomorphic with a subgroup of $\mathrm{T}_{d}$, there is a $\beta \in \Gamma$ with $\left.\beta\right|_{H}$ injective. Hence (c) $\Rightarrow$ (a) is trivial and it is enough to show that (a) $\Rightarrow$ (b) and that (b) $\Rightarrow$ (c).

(a) $\Rightarrow$ (b): Assume that (a) holds and choose a $\beta \in \Gamma$ that satisfies (a). Then there is a $v \in M(\Gamma)$ such that $\hat{v}(x)=1$ if $x \in \bar{U}$ and $\hat{v}(x)=0$ if $x \in \bar{U}+h, h \in H$ and $h \neq 0$, as was shown in the last part of the proof of Theorem 6.3. This implies that for each subset $S$ of $H$, the set $\bigcup_{h \in S}(\bar{U}+h)$ is closed, that $\alpha(\bar{U})$ is closed and that if $g \in C(\bar{U})$, then $g \circ\left(\left.\alpha\right|_{\bar{U}}\right)^{-1} \in C(\overline{\alpha(U)})$. Moreover, if $g \in C(\bar{U})$ and $P g$ denotes the function defined on $\bar{U}+H$ by $P g(x)=g(x-h)$ if $x \in \bar{U}+h$, then $P g$ is a well defined continuous function on $\bar{U}+H$. By Lemma 6.1,

$$
g \circ\left(\left.\alpha\right|_{\bar{U}}\right)^{-1} \in B(\overline{\alpha(U)})
$$

if and only if

$$
P g \in B(\bar{U}+H) \text { and }\left\|g \circ\left(\left.\alpha\right|_{\bar{U}}\right)^{-1}\right\|_{B(\overline{\alpha(U)})}=\|P g\|_{B(\bar{U}+H)} .
$$

Since $\sup _{n \in Z_{+}}\left\|\left(\beta \circ\left(\left.\alpha\right|_{\bar{U}}\right)^{-1}\right)^{n}\right\|_{B(\overline{\alpha(U))}} \leqq C$, by Parseval's identity,

$$
\forall n \in \mathbf{Z} \forall \varphi \in S(U+H):\left|\int_{G} P(n \beta)\right|_{\bar{U}}(x) \hat{\varphi}(x) d x \mid \leqq C\|\varphi\|_{\propto} .
$$

But if $\hat{\lambda} \in \Lambda$ then $\left.P(\hat{\lambda}+n \beta)\right|_{\bar{U}}(x)=\left.\langle\hat{\lambda}, x\rangle P(n \beta)\right|_{\bar{U}}(x)$ and hence, (1) also holds with $n \beta$ replaced by $\lambda+n \beta$. If $\beta Z$ denotes the subgroup of $G$ generated by $\beta$, then the annihilator of $\overline{\Lambda+\beta Z}$ is $H \cap \operatorname{Ker} \beta$. As $\left.\beta\right|_{H}$ is injective, $A+\beta Z$ is dense in $\Gamma$ and this implies by approximation that (1) holds with $n \beta$ replaced by any $\chi \in \Gamma$.

For each $\varphi, \psi \in S$ and $\chi \in \Gamma$, the function $\lambda \rightarrow \varphi(-\lambda) \psi(\lambda-\chi), \lambda \in \Lambda$, belongs to $L^{1}(\Lambda)$ and by Poisson's formula

$$
\int_{\Lambda} \varphi(-\hat{\lambda}) \psi(\hat{\imath}-\chi) d \hat{\lambda}=\int_{G}\left[\int_{H}\langle\chi, x+h\rangle \hat{\psi}(x+h) d h\right] \hat{\varphi}(x) d x
$$

(cf. for example $[11,5, \S 5])$.

Now let $\varphi \in S(U+H)$ be fixed and $K=\operatorname{supp} \hat{\varphi}$. By the compactness of $K$, there are $h_{1}, \ldots, h_{m} \in H$ such that $K \subset \bigcup_{k=1}^{m}\left(U+h_{k}\right)$. Let $K_{k}=K \cap\left(U+h_{k}\right)$ and $K^{\prime}=\bigcup_{k=1}^{m}\left(K_{k}-h_{k}\right)$, a compact subset of $U$. Choose any $\varrho_{1} \in S$ such that $\hat{\varrho}_{1}(x)=1$ if $x \in K^{\prime}$ and let $\varrho=\varrho_{1} * v$. For each $x \in K$ and $\chi \in \Gamma$ we have

$$
\left.P_{\chi}\right|_{\bar{U}}(x)=\int_{H}\langle\chi, x+h\rangle \hat{\varrho}(x+h) d h
$$

and thus, by (1) and (2),

$$
\left|\int_{\Lambda} \varphi(-\lambda) \varrho(\lambda-\chi) d \lambda\right| \leqq C\|\varphi\|_{\infty} .
$$


Since $\hat{\varrho}_{1}(x)=1$ if $x \in K^{\prime}$, for each $\psi \in S$ we have by (2)

$$
\int_{\Lambda} \varphi(-\lambda)(\psi * v)(\lambda) d \lambda=\int_{\Lambda} \varphi(-\lambda)(\psi * \varrho)(\lambda) d \lambda
$$

and as

$$
\begin{aligned}
\left|\int_{\Lambda} \varphi(-\lambda)(\psi * \varrho)(\lambda) d \lambda\right| & =\left|\int_{\Gamma} \psi(\gamma)\left[\int_{\Lambda} \varphi(-\lambda) \varrho(\lambda-\gamma) d \lambda\right] d \lambda\right| \\
& \leqq \sup _{\gamma \in \Gamma}\left|\int_{\Lambda} \varphi(-\lambda) \varrho(\lambda-\gamma) d \lambda\right| \cdot\|\psi\|_{L^{1}(\Gamma)},
\end{aligned}
$$

the proof is completed by using (3).

(b) $\Rightarrow$ (c): Suppose that (b) holds and choose a $v \in M(\Gamma)$ such that $\hat{v}(x)=1$ if $x \in \bar{U}$ and $\bar{v}(x)=0$ if $x \in \bar{U}+h, h \in H, h \neq 0$. Then if $f \in B(\bar{U})$, the function

$$
x \mapsto \int_{H} f(x+h) \hat{v}(x+h) d h \quad \text { if } x \in \bar{U}+H,
$$

is well defined, continuous and equals $f$ on $\bar{U}$. By Lemma 6.1 it is enough to show that this function is in $B(\bar{U}+H)$ and has norm at most $C\|f\|_{B(\bar{U})}$. To this end, it is sufficient by Lemma 6.2 to show that for each $\varphi \in S(U+H)$

$$
\left|\int_{G}\left[\int_{H} f(x+h) \hat{v}(x+h) d h\right] \hat{\varphi}(x) d x\right| \leqq C\|f\|_{B(U)}\|\varphi\|_{\infty} .
$$

Let $\varepsilon>0$ and choose a $\sigma \in M(\Gamma)$ such that $\hat{\sigma}(x)=f(x)$ if $x \in \bar{U}$ and such that $\|\sigma\|_{M(\Gamma)} \leqq\|f\|_{B(\bar{U})}+\varepsilon$. For $\varphi \in S(U+H)$ let $K^{\prime}$ be as in the proof of (a) $\Rightarrow$ (b) and choose $\psi \in S$ such that $\hat{\Psi}(x)=1$ if $x \in K^{\prime}$ and $\|\psi\|_{L^{1}(\Gamma)}<1+\varepsilon$. Then the function $\psi * v * \sigma \in S$ and by (2) it follows that

(5) $\int_{\Lambda} \varphi(-\lambda)(\psi * \sigma * v)(\lambda) d \lambda=\int_{G}\left[\int_{H} \hat{\sigma}(x+h) \hat{\psi}(x+h) \hat{v}(x+h) d h\right] \hat{\varphi}(x) d x$.

Since $\psi(x)=1$ if $x \in K^{\prime}$ and $\hat{\sigma}(x)=f(x)$ if $x \in \bar{U}$,

$$
\begin{aligned}
\int_{G}\left[\int_{H} \hat{\sigma}(x+h) \psi(x+h) \hat{v}(x+h) d h\right] \hat{\varphi}(x) d x \\
=\int_{G}\left[\int_{H} f(x+h) \hat{v}(x+h) d h\right] \hat{\varphi}(x) d x .
\end{aligned}
$$

By the assumption

$$
\left|\int_{\Lambda} \varphi(-\lambda)[(\psi * \sigma) * v](\lambda) d \lambda\right| \leqq C\|\varphi\|_{L^{2}(\Gamma)}\|\psi * \sigma\|_{L^{1}(\Gamma)}
$$


and as

$$
\|\psi * \sigma\|_{L^{1}(\Gamma)} \leqq\|\psi\|_{L^{1}(\Gamma)}\|\sigma\|_{M(\Gamma)} \leqq(1+\varepsilon)\left(\|f\|_{B(\bar{U})}+\varepsilon\right)
$$

and $\varepsilon>0$ was arbitrary, (4) follows from (5) and (6).

REMARK. As a result of the proof, (b) $\Leftrightarrow$ (c) for any discrete subgroup $H$ of $G$. This can be used for a proof of the weak form of Theorem 2.3 that results, when $\alpha: G_{1} \rightarrow G_{2}$ is assumed to be an epimorphism. In the earlier sections, we have only used this weak form. So suppose that $E$ is a compact subset of $G$ and that the canonical map $\alpha: G \rightarrow G / H$ is injective on a nbd $V$ of $E$. By compactness, we can find an open nbd $W$ of 0 in $G$ with $\bar{W}$ compact such that if $U=E+W$, then $\bar{U} \subset V$. Hence, there is a $\varrho \in S$ with $\varrho(x)=1$ if $x \in \bar{U}$ and supp $\hat{\varrho} \subset V$. There is also a constant $C$ such that for each $\chi \in \Gamma$

$$
\int_{\Lambda}|\varrho(\hat{\lambda}-\chi)| d \hat{\lambda} \leqq C
$$

(cf. for example $[11,5, \S 5])$. Thus, for each $\varphi \in S(U+H)$ and $\psi \in S$

$$
\left|\int_{\Lambda} \dot{\varphi}(-\hat{\lambda})(\varrho * \psi)(\hat{\lambda}) d \hat{\lambda}\right| \leqq\|\varphi\|_{L^{\alpha}(\Lambda)}\|\varrho * \psi\|_{L^{1}(\Gamma)} \leqq C\|\varphi\|_{L^{\infty} \Lambda}\|\psi\|_{L^{1}(\Gamma)},
$$

which implies that if $f \in B(E)$, then $f \circ\left(\left.\alpha\right|_{E}\right)^{-1} \in B(\alpha(E))$ as well as

$$
\left\|f \circ\left(\left.\alpha\right|_{E}\right)^{-1}\right\|_{B(\alpha(E))} \leqq C\|f\|_{B(E)} .
$$

If $\theta: W_{0} \rightarrow \mathrm{T}$ is a local character in $G=\mathrm{R}^{m} \times K$, where $K$ is compact and connected, there are nbds $W_{0}^{\prime}$ of 0 in $\mathrm{R}^{m}$ and $W_{0}^{\prime \prime}$ of 0 in $K$ and local characters $\theta^{\prime}: W_{0}^{\prime} \rightarrow \mathrm{T}$ and $\theta^{\prime \prime}: W_{0}^{\prime \prime} \rightarrow \mathrm{T}$ such that $W_{0}^{\prime} \times W_{0}^{\prime \prime} \subset W_{0}$ and $\theta(x, y)$ $=\theta^{\prime}(x) \theta^{\prime \prime}(y)$ for each $(x, y) \in W_{0}^{\prime} \times W_{0}^{\prime \prime}$. As $W_{0}^{\prime} \subset \mathrm{R}$, we may assume that $\theta^{\prime}$ is a restriction of a character. Thus the group $\tilde{G}$, constructed from $G$ and the local character $\theta$ in Theorem 2.1. is (isomorphic with) $\mathbf{R}^{m} \times \tilde{K}$, where $\tilde{K}$ is the group constructed from $K$ and the local character $\theta^{\prime \prime}$.

If $\alpha$ is the usual epimorphism of $\tilde{G}$ onto $G$, its kernel $H$ is of the form $H=\{0\}$ $\times H^{\prime \prime}$, where $H^{\prime \prime}$ is a discrete subgroup of $\tilde{K}$. Thus the annihilator $\Lambda$ of $H$ in $(\tilde{G})^{-}=R^{m} \times(\tilde{K})^{\hat{n}}$ is of the form $\Lambda=\mathrm{R}^{m} \times \Lambda^{\prime \prime}$, where $\Lambda^{\prime \prime}$ is the annihilator of $H^{\prime \prime}$ in $(\tilde{K})^{\hat{L}}$. By the corollary below, it follows that if $\tilde{U} \subset R^{m} \times \tilde{C}$, where $\tilde{C} \subset \bar{K}$ is compact and if there is a $v \in M((\tilde{G}))$ such that $\hat{v}(x)=1$ if $x \in \tilde{U}$ and $\hat{v}(x)=0$ if $x \in \widetilde{U}+h, h \in H$ and $h \neq 0$, then for each $\beta \in(G)$, the function $f=\beta \circ(\alpha \mid \tilde{U})^{-1}$ is a function with bounded powers in $B(\overline{\alpha(\tilde{U}))}$.

Corollary. Suppose that $H^{\prime}$ is a discrete subgroup of the LCA group $G^{\prime}$, let $G$ $=\mathrm{R}^{m} \times G^{\prime}$ and $H=\{0\} \times H^{\prime}$ and let $\alpha: G \rightarrow G / H$ be the canonical map. Assume 
that $U$ is an open nbd of 0 in $G$ such that for some compact subset $C^{\prime}$ in $G^{\prime}, U$ $\subset \mathrm{R}^{m} \times C^{\prime}$. If there exists a $v \in M(\Gamma)$ with $\hat{v}(x)=1$ if $x \in \bar{U}$ and $\hat{v}(x)=0$ if $x \in \bar{U}$ $+h$ for each $h \in H$ with $h \neq 0$, then there is a constant $C$ such that

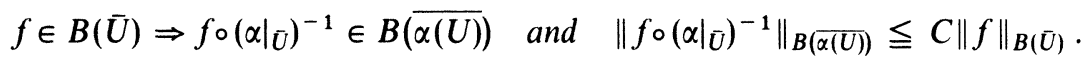

Proof. By Theorem 6.4. and (2), it is sufficient to show that if $\psi \in S$ and if $\varrho \in S$ satisfies $\varrho(x)=1$ for $x \in \operatorname{supp} \hat{\psi} \cap U$, then

$$
\int_{\Lambda}\left\lfloor * \psi * v \mid(\lambda) d \lambda \leqq C\|\psi\|_{L^{1}(\Gamma)} .\right.
$$

Since $U \subset \mathrm{R}^{m} \times C^{\prime}$, with $\psi \in S$ given, we can choose a compact subset $L$ in $\mathrm{R}^{m}$ such that supp $\hat{\psi} \cap U \subset L \times C^{\prime}$. As $C^{\prime}$ is a compact subset of $G^{\prime}$, we can choose a $\varrho_{2} \in S\left(G^{\prime}\right)$ such that $\varrho_{2}(t)=1$ if $t \in C^{\prime}$. Hence there is a constant $C_{2}$ such that for each $\gamma^{\prime} \in \Gamma^{\prime}=\left(G^{\prime}\right)^{\prime}$,

$$
\int_{\Lambda}\left|\varrho_{2}\left(\gamma^{\prime}-\hat{\imath}^{\prime}\right)\right| d \hat{\imath}^{\prime} \leqq C_{2} .
$$

As $L \subset \mathrm{R}^{m}$ is compact, we can choose a $\varrho_{1} \in S\left(\mathrm{R}^{m}\right)$ such that $\hat{\varrho}_{1}(s)=1$ if $s \in L$ and $\left\|\varrho_{1}\right\|_{L^{1}\left(\mathrm{R}^{m}\right)}<2$. Thus, as $A=\mathbf{R}^{m} \times \Lambda$, if we let $\varrho=\varrho_{1} \otimes \varrho_{2}$ then $\varrho(x)=1$ if $x \in L \times C^{\prime}$ and for each $\chi \in \Gamma, \int_{\Lambda} \varrho \varrho(\lambda-\chi) \mid d \lambda \leqq 2 C_{2}$. This implies that

$$
\int_{\Lambda}|\varrho * \psi * v| d \lambda \leqq \int_{\Gamma}\left[\int_{\Lambda}|\varrho(\lambda-\gamma)| d \lambda\right]|\psi * v|(\gamma) d \gamma \leqq 2 C_{2}\|v\|_{M(\Gamma)}\|\psi\|_{L^{1}(\Gamma)},
$$

and with $C=2 C_{2}\|v\|_{M(\Gamma)}$, the proof is complete.

\section{REFERENCES}

1. A. Beurling and H. Helson, Fourier Stieltjes transforms with bounded powers, Math. Scand. 1 (1953), 120-126.

2. N. Bourbaki, Topologie Générale, chap. 7, Eléments de Mathématique $3^{\mathrm{d}}$ ed., Hermann, Paris, 1963.

3. P. Brenner, Power bounded matrices of Fourier-Stieltjes transforms, Math. Scand. 22 (1968), 115-129; corrections Math. Scand. 30 (1972), 150-151.

4. P. J. Cohen, On homomorphisms of group algebras, Amer. J. Math. 82 (1960), 213-226.

5. K. de Leeuw and C. S. Herz, An invariance property of spectral synthesis, Illinois J. Math. 9 (1965), 220-229.

6. Y. Domar, A theorem of Beurling-Helson type, Math. Scand. 33 (1973), 139-144.

7. C. S. Herz, The spectral theory of bounded functions, Trans. Amer. Math. Soc. 94 (1960), 181232.

8. E. Hewitt and K. A. Ross, Abstract harmonic analysis, I (Grundlehren Math. Wissensch. 115), Springer-Verlag, Berlin - Heidelberg - New York, 1963.

9. Y. Meyer, Algebraic numbers and harmonic analysis, North-Holland, Amsterdam, 1972.

10. L. S. Pontryagin, Topological groups, $2^{\text {nd }}$ ed., Gordon and Breach, New York, 1966. 
11. H. Reiter, Classical harmonic analysis and locally compact groups, Clarendon Press, Oxford, 1968.

12. W. Rudin, Fourier analysis on groups, Interscience, New York, 1960.

13. E. Svensson, A note on power bounded restrictions of Fourier-Stieltjes transforms, Math. Scand. 46 (1980), 154-156.

DEPARTMENT OF MATHEMATICS

UNIVERSITY OF GÖTEBORG AND CTH

S-412 96 GÖTEBORG, SWEDEN 